\title{
Reward pretraining and discrimination learning set*
}

\author{
DOUGLAS L. MEDIN \\ The Rockefeller University, New York, New York 10021
}

\begin{abstract}
Pigtailed monkeys were trained on discrimination problems involving objects that had been previously paired with reward, previously paired with nonreward, or objects not associated with any pretraining. In two separate studies, no differences on these various problem formats were observed, but performance improved considerably with practice. These results are contrary to the theories for learning set formation of Spence and Medin.
\end{abstract}

When monkeys are given a series of object discriminations, their performance on new problems gets better and better. Early in the series, they make many errors but, after extensive practice, learning takes place after just a few errors. A major theoretical problem is to specify the source of improvement in discrimination performance.

The present study tests one implication of some theories for learning set formation. The basic procedure involves pairing objects with reward or nonreward during pretraining, followed by discrimination training where two previously rewarded objects are paired and two previously nonrewarded objects are paired. The variable of main interest is the relative rate of learning these two kinds of problems. First, it is in order to review the theories making a clear prediction concerning this paradigm.

Although it is certainly not a well-publicized fact, Spence's (1936) original theory of discrimination learning predicts that learning to learn will occur. The most extensive discussion of the application of Spence's theory to learning set is given by Reese (1964). Space does not permit a detailed review of Spence's theory, but it is important to bring out the facets of the theory which underlie improvement on object discriminations. These main factors are: (1) the initial strengths associated with the two objects of a discrimination problem, (2) the difference in these initial strengths, which provides a basis for stimulus preferences, and (3) the difference in strengths of irrelevant cues which must be overcome before a probelm is solved. We shall consider these in turn.

Initial strengths alter performance through their influence on the effectiveness of reward and nonreward. The effectiveness of a reward first increases with strength to a maximum and then, for high strengths, decreases once more. The change in strength produced by a nonreward increases linearly with strength. Overall then, changes in strength are greatest when strengths are

*This research was supported by United States Public Health Service Grants MH25134 and MH16100. in the middle to high range. When the preferred stimulus is incorrect, stimulus preferences can serve to retard learning. As a consequence, decreases in initial stimulus preferences facilitate learning. Spence's original paper illustrates the influence of irrelevant cues, such as spatial or position habits, on learning. Decreases in these strength differences are also associated with faster learning.

To assess predictions of Spence's theory concerning the effects of pretraining with rewards, computer simulations of these pretraining conditions were run, along with variations of the three factors underlying learning set formation just discussed. That is, the initial parameter values for the various simulations systematically varied the initial strengths and strength differences for the relevant object cues and the initial strengths and strength differences for irrelevant spatial cues. For a given set of initial values, simulations of the pretraining conditions were run, using the same parameter values for the effects of reward and nonreward that Spence employed in his 1936 paper. The habit strengths for the cues after pretraining were then used as input values for a simulation of discrimination training. One hundred statistical Ss were run for each combination of conditions. These simulations indicated that the initial strength of object cues was the main factor responsible for faster learning, with the other two factors contributing less, and about equally, to learning set formation. Except in the range of very low strengths and strong stimulus preferences, the simulations further showed that pairing objects with rewards during pretraining should strongly facilitate later learning, relative to no pretraining and that pairing objects with nonreward might have a small negative effect on learning relative to no pretraining.

A theory which this author recently proposed (Medin, 1972) makes the same prediction as Spence's theory, but for different reasons. According to this theory, the main source of faster learning is that object cues come to have greater strength or feedback value than other cues. In effect, if the two object cues are high enough in strength, relative to other cues, almost all choices will be controlled by object cues. Objects associated with 
reward should have high strength, relative to other cues, and discriminations should be solved quickly. Objects associated with nonreward should be low in strength, relative to other cues, which should retard object-discrimination learning.

In the experiments to be reported, pretraining was always given with four objects, two of which were associated with reward while the other two were paired with nonreward. After this pretraining, the two previously rewarded objects were paired to create one discrimination problem, and the two previously nonrewarded objects were paired to create another discrimination problem. Additional comparisons and conditions were included to insure that the pretraining was effective. According to the theories, the rewarded pretraining should produce faster learning than nonrewarded pretraining. In one related study, Warren and Sinha (1959) found that pretraining consisting of pairing both the positive and negative stimuli with reward, produced a small, but reliable, facilitation on pattern-discrimination learning compared with control conditions with no pretraining.

Since the character of this study is somewhat out of line with more usual studies of pretraining effects, some cautionary remarks are in order. First, the main concern is to assess the adequacy of theories purporting to account for learning-to-learn effects in monkeys, and pretraining effects are a convenient means to this end. There is an extensive literature on pretraining effects conducted by researchers such as Amsel, Bitterman, Forgus, and the Gibsons (see Sutherland \& Mackintosh, 1971 , for a review) but the present study has, at best, peripheral relevance to this line of work. To begin with, monkeys tested on learning set problems usually have received extensive pretraining (the present study is no exception) and are, in no sense, naive. Learning set sophistication in monkeys is traditionally defined in terms of Trial 2 performance on problems rather than the number of problems or trials on which monkeys have received training. Therefore, the presence or absence of pretraining effects in the present study will have essentially no relevance for theories concerning pretraining effects in naive Ss. However, the presence or absence of pretraining effects is directly relevant to the learning-to-learn theories of Spence and Medin. That is, if these theories are to represent viable explanations of learning set formation in monkeys, then they will be led to expect pretraining effects, even in quite experienced animals, since the same explanatory principle is to be invoked to account for both pretraining effects and improvements in learning.

\section{EXPERIMENT I}

The first experiment compared the effects of pretraining with rewards and nonrewards on discrimination learning given immediately thereafter.
Response latencies were recorded for both pretraining and discrimination learning.

\section{Method}

Subjects. The Ss were eight jungle-born pigtailed monkeys, Macaca nemestrina, 3-4 years old. All animals had received approximately 6 months of training on various discriminations, including simultaneous object discriminations. At the start of this experiment, their performance was in the range of $60 \%$ correct on Object Discrimination Trials 2-6.

Apparatus. A Wisconsin General Test Apparatus (WGTA) was used with a gray formboard. The foodwells of the food tray were spaced $30 \mathrm{~cm}$ apart, edge-to-edge. Photocells were imbedded in opposite sides of the food tray and just in front of the foodwells. Opening the opaque door of the WGTA released a microswitch initiating a timer, which ran until the photobeam was broken as the monkeys displaced a stimulus object. The 120 stimulus objects for the experiment were selected from a pool of common-use and manufactured objects.

Procedure. Each day of training consisted of 16 single-choice pretraining trials, followed by 24 two-choice discrimination learning trials. Four new objects were used each day. Each of these objects, which shall be designated as A, B, C, and D, appeared four times during pretraining, twice on the left and twice on the right. The assignment of $\mathrm{A}, \mathrm{B}, \mathrm{C}$, and D to the objects was randomized for each $\mathrm{S}$, but the monkeys saw the same object on a day.

After the opaque screen was raised to initiate a trial, the $\mathrm{S}$ would displace the object and either find or fail to find a raisin reward in the foodwell. The food tray was withdrawn and the trial terminated if the $\mathrm{S}$ did not respond within $15 \mathrm{sec}$. The A and $B$ objects were paired with reward and the $\mathrm{C}$ and $\mathrm{D}$ objects were paired with nonreward on these trials. The order of the A, $\mathrm{B}, \mathrm{C}$, and $\mathrm{D}$ object trials was randomized. The intertrial interval was approximately $12 \mathrm{sec}$.

Discrimination training immediately followed pretraining. The rewarded $A$ and $B$ objects were paired to create one discrimination and the $C$ and $D$ objects were paired for the second discrimination. Arbitrarily, the $\mathrm{A}$ and $\mathrm{C}$ objects were designated as correct on these discriminations. Twelve trials, randomly intermixed, were given on each of these two problems. The position of the rewarded object was random subject to the constraint that no more than four consecutive trials had the same position correct. A noncorrection procedure was used and correct responses were rewarded with a raisin. Again, response latencies were recorded.

Testing continued 5 days/week, for 6 weeks. Midway through the experiment, four special test trials were added. For these trials, which immediately followed discrimination training, the A and $C$ objects were paired and the B and D objects were paired. Each of these pairings appeared twice, with position counterbalanced, and all responses were rewarded. These tests were designed so that it could be determined whether or not the pretraining effects persisted after discrimination training.

\section{Results}

Pretraining. Response latencies on the pretraining trials are given in Table 1. By the fourth presentation, there were large differences in latency, depending upon whether reward or nonreward would be forthcoming. Every animal showed this effect, which was statistically reliable $(t=3.99$, df $=7, p<.01$, using the mean latency over the 30 days for each animal). The lack of difference on the first presentation confirms that the pretraining procedure produced the differences in latency.

Discrimination Training. The latency effects persisted 
into discrimination testing. The average latency on the first $\mathrm{AB}$ trial was $1.62 \mathrm{sec}$ compared to $2.00 \mathrm{sec}$ for the initial CD trial. All but one monkey showed this difference, and it proved to be statistically significant $(\mathrm{t}=3.18, \mathrm{df}=7, \mathrm{p}<.02)$.

Learning improved over the 30 days of testing, from a mean of 4.09 errors/problem, in the first 10 days, to a mean of 3.43 errors/problem, for the last 10 days. The pretraining conditions produced no differences at all in learning. Ss averaged 3.62 errors on $A B$ problems and 3.60 errors on $C D$ problems. An analysis of variance indicated that effect of 10-day blocks of practice was significant $(F=5.40, d f=2 / 14, p<.05)$, but no other effect or interaction approached significance.

Choice Tests. The pretraining effects continued to be manifest on the nondifferentially rewarded AC, BD choice tests. The A objects were chosen over the $\mathrm{C}$ objects on $65.2 \%$ of these trials and B objects were chosen over D objects on $67.0 \%$ of these trials. Both trends were observed in all animals and were highly reliable statistically.

\section{EXPERIMENT II}

Although the pretraining conditions were effective in altering response latency and produced preferences which persisted beyond discrimination training, this same pretraining produced no differences in learning efficiency. The second experiment introduced a 24-h delay between pretraining and discrimination training to see if short-term factors associated with pretraining might be masking pretraining effects on learning. In addition, problems with objects associated with no pretraining were included.

\section{Method}

Subjects. The Ss were six of the eight pigtailed monkeys tested in the first experiment. The other two monkeys were not used for reasons unrelated to either of the experiments.

Apparatus. The same WGTA and other details of apparatus used in the first experiment were again used. An additional set of 168 junk objects was used for the various stimulus conditions.

Procedure. The general plan was the same as in Experiment I. For preliminary training, the same procedure of pairing $A$ and $B$ objects with reward and $C$ and $D$ objects with nonreward was used without modification.

Discrimination training was different in several ways. Both $A B$ and $C D$ discriminations were given with $A$ and $C$ correct as before, but the A, B, C, and D objects for discrimination training were the set of objects used in preliminary training on the preceding, rather than the current, day. For the first day, this was, of course, impossible, and a randomly selected set of four objects served as A, B, C, and D. In addition, four new objects, designated $\mathrm{E}, \mathrm{F}, \mathrm{G}$, and $\mathrm{H}$, were introduced each day in order to create two new discrimination problems $(\mathrm{E}+\mathrm{F}-$ and $\mathrm{G}+\mathrm{H}-$-). Six trials were given on each of these four discriminations. The $A B$ and $\mathrm{CD}$ problems were randomly intermixed and the $\mathrm{EF}$ and $\mathrm{GH}$ problems were randomly intermixed. Following preliminary training, 12 trials on one of these pairs of discriminations were followed by 12 trials on the other pair of discriminations, with the particular order being randomly determined.
Table 1

Response Latency in Seconds as a Function of Presentations for Objects Paired With Reward and Nonreward

\begin{tabular}{|c|c|c|c|c|c|}
\hline & & \multicolumn{4}{|c|}{ Presentation } \\
\hline & & 1 & 2 & 3 & 4 \\
\hline$A, B$ & (Rewarded) & 2.10 & 1.76 & 1.73 & 1.60 \\
\hline C, D & (Not Rewarded) & 2.09 & 1.66 & 2.21 & 2.18 \\
\hline
\end{tabular}

After discrimination training, Ss were again given nondifferentially rewarded AC and BD tests involving the objects that were used in that day's discrimination training (and the preceding day's preliminary testing). Two tests, counterbalanced for position, were given on each pair of objects.

The experiment followed the first experiment without any break and continued for 21 days. Performance on the initial day was not included because the conditions involving A, B, C, and D objects with training on the preceding day were not satisfied.

\section{Results}

Preliminary Training. The pretraining reward conditions produced the expected latency differences. By the fourth presentation, the monkeys' latencies averaged $1.11 \mathrm{sec}$ for $\mathrm{A}$ and $\mathrm{B}$ objects and $1.91 \mathrm{sec}$ for $\mathrm{C}$ and $\mathrm{D}$ objects. Every $\mathrm{S}$ showed this effect and it was statistically significant $(\mathrm{t}=3.49, \mathrm{df}=5, \mathrm{p}<.05$, using mean latency for each animal). Clearly, the presence or absence of reward was associated with the stimulus objects.

Discrimination Training. The latency difference between the first $A B$ trial and the first $C D$ trial observed in Experiment $I$ was not observed when these trials followed after $24 \mathrm{~h}$ and considerable intervening experience. The monkeys were actually slightly faster on the initial $E F$ and $\mathrm{GH}$ trials than on the $\mathrm{AB}$ and $\mathrm{CD}$ trials, but this effect was small and not reliable.

Discrimination learning again improved with 10-day blocks of practice, from an average of 2.14 errors/problem to an average of 1.89 errors/six-trial problem. Ss averaged 2.02 errors on the AB problems, 2.05 errors on DC problems, and 2.03 errors on EF and GH problems. An analysis of variance indicated that only blocks of practice was statistically reliable $(F=$ $9.19, \mathrm{df}=1 / 5, \mathrm{p}<.05)$.

The choice tests given at the end of each day's training indicated that the pretraining effects did persist over the 24-h period between pretraining and discrimination testing. For the $\mathrm{AC}$ test, A objects were chosen $65.8 \%$ of the time, and for the BD tests, B was chosen $67.9 \%$ of the time. All monkeys displayed these effects, which were statistically significant beyond the .01 level.

\section{GENERAL DISCUSSION}

In both experiments, pretraining with reward and nonreward had persistent effects on response latency 
and object choices. These results indicate that the basic manipulation which was supposed to produce differences in learning rate was satisfied. Yet, in spite of the power provided by 50 days of testing, none of the predicted differences in learning was observed.

Medin's theory predicted that pretraining with rewards would facilitate learning and pretraining with nonreward would impair learning, relative to control problems with no pretraining. Neither of these anticipated effects were observed. It is possible that a model more kindred to attention theory might have more success in accounting for learning set formation. Sutherland and Mackintosh (1971) argued, on both theoretical and empirical grounds, that nondifferential reward should not alter the strength of an analyzer, so such a theory would predict that pretraining would have no effect.

An alternative possibility is that the strengths of the various cues in a problem are not independent. The design of the experiment carries the assumption that irrelevant cues such as position will be intermediate between the rewarded and nonrewarded objects since they are only associated with reward on half of the pretraining trials. However, if the strength of, say, a position cue depends upon its context (e.g., whether or not A or B objects or C or D objects cover the foodweil), then the pretraining procedure would fail to place $A$ and $B$ objects at an advantage in comparison to irrelevant cues. There exists direct evidence for the expectation that the various cues are not independent (e.g., Spiker, 1963; Flagg \& Medin, 1973), and theories of discrimination learning embodying this assumption have been advanced (Spiker, 1963, 1970; Medin, 1973). Spiker's theory represents one way of modifying Hull-Spence discrimination theory to account for the present results.

As it is, Spence's theory incorrectly predicts that pairing the stimulus objects with reward should improve learning. One might offer that the initial strength associated with the objects was so small that differences either would not be expected or were masked. For this suggestion to be given credence, performance would have to be very poor and improve only modestly with practice. But this was not the case. If one traces the performance of the six monkeys completing both experiments, percentage correct on problems of Trials 2.6 increases from 60.1 , on the first block, to 77.8 , on the fifth 10-day block of testing.

In summary, the present study suggests that pairing stimulus objects with rewards and nonrewards does not mimic separate stages in learning set formation, contrary to the theories of Spence and Medin. These results from one small group of monkeys should not be given excessive weight but, since the conditions which should have produced pretraining effects were met, the present experiments must be viewed as questioning the appropriateness of these theories in accounting for learning set formation in monkeys.

\section{REFERENCES}

Flagg, S. F., \& Medin, D. L. Constant irrelevant cues and stimulus generalization in monkeys. Jo urnal of Comparative \& Physiological Psychology, 1973, 85, 339-345.

Medin, D. L. Role of reinforcement in diserimination learning set in monkeys. Psychological Bulletin, 1972, 77, 305-318.

Medin, D. L. Context in primate discrimination learning. Paper presented at Psychonomic Society meetings, St. Louis, 1973.

Reese, H, W. Discrimination learning set in rhesus monkeys. Psychological Bulletin, 1964, 61, 321-340.

Spence, $K$. W. The nature of discrimination learning in animals. Psychological R eview, 1936, 43, 421-449.

Spiker, C. C. The hypothesis of stimulus interaction and an explanation of stimulus compounding. In L. P. Lipsitt and C. C. Spiker (Eds.), Advances in child development and behavior. Vol. 1. New York: Academic Press, 1963.

Spiker, C. C. An extension of Hull-Spence discrimination learning theory. Psychological R eview, 1970, 77, 496-515.

Sutherland, N. S., \& Mackintosh, N. J. Mechanisms of animal discrimination learning. New York: Academic Press, 1971.

Warren, J, M., \& Sinha, M. M. Interaction between learning sets in monkeys. Journal of Genetic Psychology, 1959, 95, 19-25.

(R eceived for publication April 29, 1974; revision received June $17,1974$. ) 